\title{
Article \\ Engineering Au Nanoclusters for Relay Luminescence Enhancement with Aggregation-Induced Emission
}

\author{
Pei Zhou ${ }^{1,2, *(\mathbb{D})}$, Nirmal Goswami $\left.{ }^{3} \mathbb{(}\right)$, Tiankai Chen ${ }^{4}$, Xiaoman Liu ${ }^{2}$ and Xin Huang ${ }^{2, *}$ \\ 1 School of Environmental and Municipal Engineering, North China University of Water Resources and Electric \\ Power, Collaborative Innovation Center for Efficient Utilization of Water Resources, Henan Key Laboratory of \\ Water Environment Simulation and Treatment, Zhengzhou 450046, China \\ 2 MIIT Key Laboratory of Critical Materials Technology for New Energy Conversion and Storage, School of \\ Chemistry and Chemical Engineering, Harbin Institute of Technology, Harbin 150001, China; \\ liuxiaoman@hit.edu.cn \\ 3 Materials Chemistry Department, CSIR-Institute of Minerals and Materials Technology, Acharya Vihar, \\ Bhubaneswar 751013, India; ngoswami@immt.res.in \\ 4 Department of Chemical and Biomolecular Engineering, National University of Singapore, 10 Kent Ridge \\ Crescent, Singapore 119260, Singapore; chen.tiankai@u.nus.edu \\ * Correspondence: peizhou@ncwu.edu.cn (P.Z.); xinhuang@hit.edu.cn (X.H.)
}

check for updates

Citation: Zhou, P.; Goswami, N.;

Chen, T.; Liu, X.; Huang, X.

Engineering Au Nanoclusters for Relay Luminescence Enhancement with Aggregation-Induced Emission. Nanomaterials 2022, 12, 777. https:// doi.org/10.3390/nano12050777

Academic Editors: Rodolphe Antoine, Qiaofeng Yao, Xun Yuan, Zhennan Wu and Marcin Runowski

Received: 16 December 2021

Accepted: 17 February 2022

Published: 25 February 2022

Publisher's Note: MDPI stays neutral with regard to jurisdictional claims in published maps and institutional affiliations.

Copyright: () 2022 by the authors. Licensee MDPI, Basel, Switzerland. This article is an open access article distributed under the terms and conditions of the Creative Commons Attribution (CC BY) license (https:// creativecommons.org/licenses/by/ $4.0 /)$.

\begin{abstract}
The research of aggregation-induced emission (AIE) has been growing rapidly for the design of highly luminescent materials, as exemplified by the library of AIE-active materials (or AIEgens) fabricated and explored for diverse applications in different fields. Herein, we reported a relay luminescence enhancement of luminescent Au nanoclusters (Au NCs) through AIE. In addition, we demonstrated the emergence of reduced aggregation-caused luminescence by adjusting the temperature of the Au NC solution. The key to induce this effect is to attach a thermosensitive polymer poly(N-isopropylacrylamide) (PNIPAAm) on the surface of Au NCs, which will shrink at high temperature. More interestingly, the as-synthesized Au NCs-PNIPAAm can self-assemble into vesicles, resulting in an obvious decrease in the luminescence intensity in aqueous solution. The combination of relay luminescence enhancement (by AIE) and luminescence decrease (induced by thermosensitive polymers) will be beneficial to the understanding and manipulation of the optical properties of Au NCs, paving the way for their practical applications.
\end{abstract}

Keywords: luminescent gold nanoclusters; aggregation-induced emission; relay luminescence enhancement; thermosensitive polymer; self-assembly

\section{Introduction}

Thiolate-protected gold nanoclusters ( $\mathrm{Au} \mathrm{NCs,}<2 \mathrm{~nm}$ ) have witnessed dramatic growth in both basic research and practical applications [1-5], mainly due to their unique physical and chemical properties, such as HOMO-LUMO transitions, quantized charging, molecular magnetism, molecular chirality, and photoluminescence [6-9]. In particular, the fabrication of luminescent Au NCs has become an interest of research in the NC community as $\mathrm{Au}$ NCs not only show strong luminescence but also feature low toxicity and good biocompatibility [10-15]. Due to the intriguing physicochemical properties, luminescent $\mathrm{Au}$ NCs find increasing acceptance in various applications in sensing (as luminescent probes for chemical sensors and biosensors), bioimaging, and light-emitting diodes (LEDs) [16-22]. Luminescent Au NCs are usually synthesized by reducing Au(I)-thiolate complexes with strong reducing agents, such as sodium borohydride, $\mathrm{NaBH}_{4}$. However, these $\mathrm{Au} \mathrm{NCs}$ often exhibit weak luminescence and their quantum yields (QYs) rarely exceed 0.1\% [23]. A technical challenge in this field is therefore to synthesize Au NCs with strong luminescence, which has been partially addressed by some recent studies in developing efficient synthesis methods for luminescent Au NCs [12,24-34]. 
For example, our group developed a thermal reduction method to produce thiolateprotected Au NCs with a QY of $\sim 15 \%$ [35]. The strong luminescence of the thiolate-protected $\mathrm{Au} \mathrm{NCs}$ is attributed to the aggregation of $\mathrm{Au}(\mathrm{I})$-thiolate complexes onto the $\mathrm{Au}(0)$ core. This aggregation-induced emission (AIE) phenomenon is constructive, because when aggregated in solution, non-luminescent molecules can generate strong luminescence, and the degree of aggregation can be used to control the intensity of luminescence [36,37]. Importantly, the implementation of AIE effect on cluster luminescence is rather a recent entry in the NC field. In addition, recently our group and several other groups identified some AIE-type metal NCs, most of which show a higher QY than the conventional thiolate-protected Au NCs [38-41]. In fact, the two key factors leading to the AIE effect are restriction of intramolecular rotation (RIR) and intramolecular vibration (RIV), which are well-demonstrated in organic AIEgens [42-44], and they are also applicable to thiolateprotected Au NCs. Although recent progress has been made in the synthesis of AIE-type $\mathrm{Au}$ NCs, the mechanism of the AIE effect on NC luminescence is not yet fully understood. In addition, it is still challenging to finely control the luminescence properties of AIE-type metal NCs by modifying their surface structure.

Generally, AIE-active molecules consist of a number of free rotors and vibrators, such as phenyl in a typical propeller-like non-planar molecule, hexaphenylsilole (HPS) [28,29]. Once the rotation and vibration of these rotors and vibrators are greatly restricted, that is, they are unable to rotate or vibrate freely in a dilute solution, a strong AIE effect will occur. The aggregation behavior can be induced by several physical, chemical, and engineering strategies. For example, temperature or pressure can be used to construct the aggregated materials through physical strategies [45-47]. In a typical chemical strategy, weakly polar solvents can be used to adjust the viscosity and polarity of the solution. By applying these strategies, various AIE materials have been successfully designed for applications in bioimaging [48-50], biomedicine [51], and sensors [52]. In another type of luminescent material, the luminescence intensity can also be reduced at high concentrations (forming aggregates), which is attributed to a distinctly different mechanism called aggregation-caused quenching (ACQ) [44]. Many conventional luminescent molecules and luminophores, such as planar luminophoric molecules and quantum dots [53,54], inherit this ACQ effect [55]. In view of the expected AIE and undesirable ACQ effects, we hypothesized that luminescent $\mathrm{Au}$ NCs instead of conventional non-luminescent molecules can contribute relay luminescence enhancement toward the AIE effect, which may be of interest to the undiscovered optical properties of metal NCs. Researchers from various fields are motivated to further decipher the AIE mechanisms [56,57]. More interestingly, by attaching polymer poly(N-isopropylacrylamide (PNIPAAm) as an additional ligand to the Au NC surface, the as-grafted thermosensitive polymers can be designed to shrink at high temperature, which may affect the luminescence of the AIE-type Au NCs. Another interesting question is whether the as-generated Au NCs-PNIPAAm can also show relay intensity decrease after the relay luminescence enhancement (due to the AIE effect). Exposing this multifunctional $\mathrm{Au}$ NC derivative in a programmed relayed mode will further encourage us to understand the undiscovered optical properties of Au NCs.

Under the guidance of the design strategy of relay enhancement with AIE characteristics and decrease of luminescence intensity with temperature, we can now control the aggregation of $\mathrm{Au}(\mathrm{I})-\mathrm{SG}$ (SG denotes glutathione) complexes and PNIPAAm to generate relay luminescence enhancement. We first prepared a luminescent $\mathrm{Au} N \mathrm{NC}$ that exhibits strong luminescence at $610 \mathrm{~nm}\left(\lambda_{\mathrm{ex}}=365 \mathrm{~nm}\right)$ with a QY of $\sim 15 \%$. Subsequently, PNIPAAm was used to construct Au NCs-PNIPAAm conjugates, where an end-capped mercaptothiazolineactivated PNIPAAm reacted with the primary amine group of GSH on the surface of Au NCs. The resulting conjugates showed a strong relay luminescence enhancement, which is much higher than the original luminescent $\mathrm{Au}$ NCs without aggregation at room temperature. However, above the lower critical solution temperature (LCST) of the thermosensitive polymer (e.g., $32{ }^{\circ} \mathrm{C}$ ), the solution showed weak luminescence, which was induced by the aggregation of the Au NCs-PNIPAAm conjugates at a high temperature. Therefore, 
we attribute the strong relay enhancement in luminescence of the NC conjugates in water to the restriction of RIR and RIV associated with the extended PNIPAAm chains in the non-aggregated state. In addition, the well-defined Au NCs-PNIPAAm conjugates can self-assemble into vesicles in aqueous solution, which can guide the transition from AIE to relay luminescence intensity decrease by adjusting the solution temperature (Scheme 1). These two aspects related to the luminescence of $\mathrm{Au}$ NCs provide a good platform to design high-quality luminescent metal NCs.

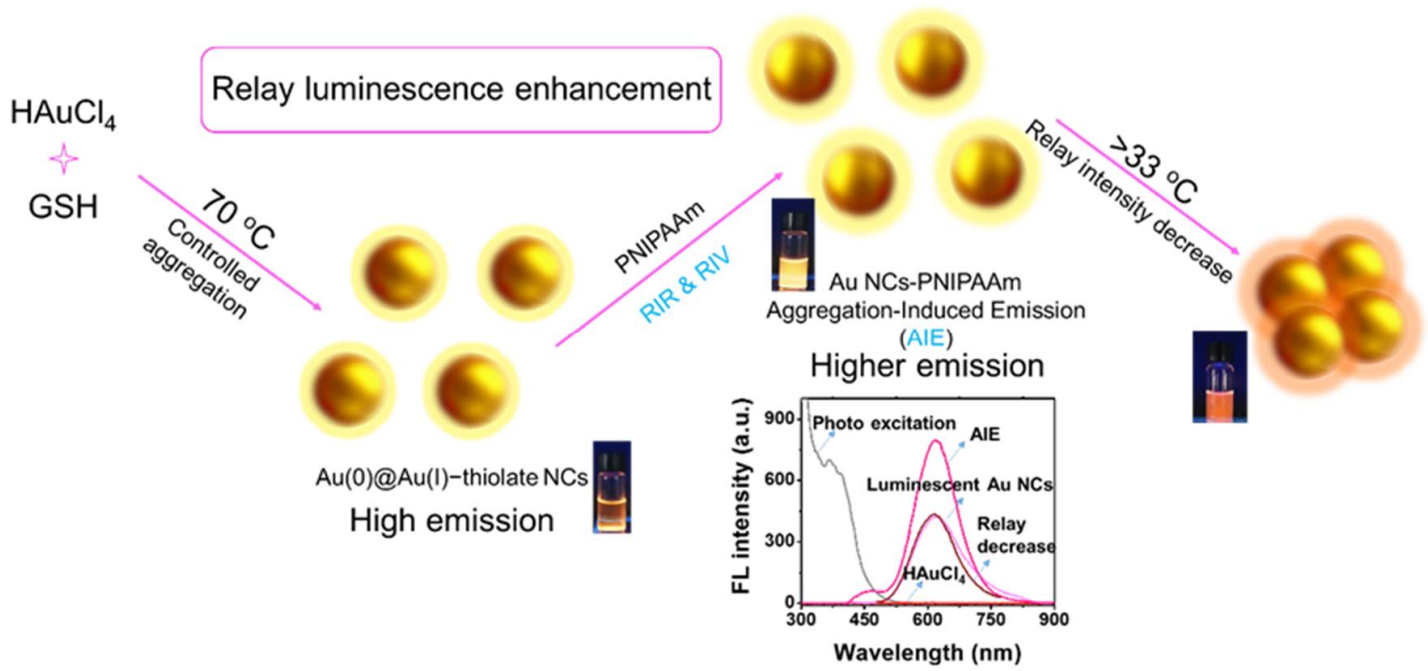

Scheme 1. Schematic illustration of the synthesis of luminescent Au NCs and Au NCs-PNIPAAm conjugates, showing the relay luminescence enhancement with AIE characteristics and relay intensity decrease with thermosensitive properties.

\section{Experimental Materials and Methods}

\subsection{Materials}

Hydrogen tetrachloroaurate trihydrate $\left(\mathrm{HAuCl}_{4} \cdot 3 \mathrm{H}_{2} \mathrm{O}\right)$ and L-glutathione in the reduced form (GSH) were obtained from Sigma-Aldrich. N-isopropylacrylamide (NIPAAm, Aldrich, St. Louis, MO, USA, 98\%) was recrystallized twice in hexane and toluene prior to use. $2,2^{\prime}$ Azobis-(isobutyronitrile) (AIBN, Sigma, St. Louis, MO, USA, 98\%) was recrystallized from methanol. 2-mercaptothiazoline (Sigma, 98\%), acetonitrile $\left(\mathrm{CH}_{3} \mathrm{CN}, 99.5 \%\right.$, Aladdin Industrial Corporation, Shangai, China), and glutaraldehyde (GA, Aladdin, AR, Shangai, China) were used as received. Ultrapure water (Milli-Q, Millipore, Burlington, MA, USA) with a resistivity of $18.2 \mathrm{M} \Omega$ was used as the solvent throughout the study. All other reagents and solvents are of analytical grade and can be used directly without further purification.

\subsection{Characterization Methods}

UV-visible absorption spectra were recorded using a Shimadzu UV-1800 photospectrometer (Shimadzu, Kyoto, Japan). Experiments were carried out in a $1.5 \mathrm{~mL}$ semimicro quartz cuvette with a $1 \mathrm{~cm}$ optical path. Fluorescence spectra were recorded on a PerkinElmer LS-55 fluorescence spectrometer (PerkinElmer, Waltham, MA, USA). Surface potentials of $\mathrm{Au}$ NCs and $\mathrm{Au}$ NCs-PNIPAAm were measured in aqueous solutions on a Malvern Zetasizer (Zeta) Nano-ZS equipment (Malvern Instruments, Malvern, UK), and $\zeta$-potential was obtained using the Smoluchowski relation. The average particle size and size distribution of the NCs $\left(0.2 \mathrm{mg} \mathrm{mL}^{-1}, \mathrm{pH}\right.$ 6.8) were characterized by dynamic light scattering (DLS) on a Malvern Zetasizer Nano-ZS at a fixed scattering angle of $90^{\circ}$, after being filtered by $0.45 \mu \mathrm{m}$ Millipore filters. ${ }^{1} \mathrm{H}$ NMR spectra were recorded on Bruker Advance- $400 \mathrm{MHz}$ spectrometer (Bruker Corporation, Ettlingen, Germany), with $\mathrm{CDCl}_{3}$ as solvent at room temperature. Chemical shifts $(\delta)$ were expressed in ppm. X-ray photoelectron spectroscopy (XPS) measurements were performed on a VG Escalab MKII spectrometer (VG Scientific, Waltham, MA, USA). Thermogravimetric analysis (TGA) was conducted 
on a Shimadzu TGA-60 analyzer (Shimadzu, Japan) under $\mathrm{N}_{2}$ atmosphere (flow rate of $20 \mathrm{~mL} \mathrm{~min}^{-1}$ ). Photoluminescence lifetimes were measured by time-correlated singlephoton counting (TCSPC) on a Horiba Jobin Yvon Fluorolog-3 spectrofluorometer (Horiba Scientific, Kyoto, Japan) with a pulsed light-emitting diode (LED) (344 nm, pulse duration $<1 \mathrm{~ns})$ as the excitation source. Optical and fluorescence microscopy was performed on a Nikon TS100 manual inverted fluorescence microscope at 100× magnification (Nikon, Tokyo, Japan). Confocal laser scanning microscope (CLSM) images were obtained with a Nikon A1 laser scanning microscope equipped with $100 \times$ oil immersion objective. A drop of hollow vesicles suspension was added to Lab-Tek chamber (Electron Microscopy Sciences, Hatfield, PA, USA) (Nikon, Japan), which was then filled with ultrapure water, and the vesicles were allowed to settle down. Transmission electron microscopy (TEM) images were taken on a JEOL JEM 2010 microscope operating at $200 \mathrm{kV}$. Atomic force microscopy (AFM) images were obtained using a Bruker Dimension ICON (JEOL, Tokyo, Japan). Silicon wafers were cleaned with DI water and dried with a stream of nitrogen. Thickness and roughness of the vesicles were determined as half of the height of the collapsed flat regions of dried vesicles from the height histograms using NanoScope analysis software (Bruker Corporation, Germany). The $\mathrm{pH}$ value was measured using METTLER TOLEDO Seven Compact meter (Mettler-Toledo, Greifensee, Switzerland).

\subsection{Synthesis of Luminescent $A u$ NCs}

Freshly prepared aqueous solutions of $\mathrm{HAuCl}_{4}(20 \mathrm{mM}, 1 \mathrm{~mL})$ and $\mathrm{GSH}(100 \mathrm{mM}$, $0.3 \mathrm{~mL}$ ) were mixed with $8.7 \mathrm{~mL}$ of ultrapure water at $25^{\circ} \mathrm{C}$. The reaction mixture was heated to $70^{\circ} \mathrm{C}$ under gentle stirring $(500 \mathrm{rpm})$ for $24 \mathrm{~h}$. An aqueous solution of strongly orange-emitting Au NCs was obtained.

\subsection{Synthesis of End-Capped Mercaptothiazoline-Activated PNIPAAm by RAFT Polymerization}

The modification of mercaptothiazoline-activated activated PNIPAAm was according to the reported method. For the mercaptothiazoline activation, briefly, mercaptothiazolineactivated trithiol-RAFT agent $(18.9 \mathrm{mg}, 50 \mu \mathrm{mol})$, AIBN $(1.7 \mathrm{mg}, 10 \mu \mathrm{mol})$, NIPAAm $(847 \mathrm{mg}, 7.5 \mathrm{mmol})$, and acetonitrile $(8 \mathrm{~mL})$ were added to a $25 \mathrm{~mL}$ of round-bottom flask. The flask was then sealed and the solution was degassed via four freeze pump-thaw cycles. The polymerization was carried out at $60^{\circ} \mathrm{C}$ for $8 \mathrm{~h}$ (conversion $45 \%$ ), and the resulting product was purified by three times precipitation in diethyl ether/hexane (2:1 volume ratio). The as-obtained polymer was characterized by ${ }^{1} \mathrm{H}$ NMR spectroscopy in $\mathrm{CDCl}_{3}$. The proton signal $(\delta, 3.3 \mathrm{ppm})$ from the mercaptothiazoline at the end of the PNIPAAm chain was visible in the ${ }^{1} \mathrm{H}$ NMR spectrum. The molecular weight of the as-obtained PNIPAAm was determined by ${ }^{1} \mathrm{H}$ NMR through comparing the integral of the proton of the $\mathrm{CH}$ signal at $\delta=4.01 \mathrm{ppm}$ in the repeat unit of NIPAAm $\left(\mathrm{Mn} 9600 \mathrm{~g} \mathrm{~mol}^{-1}\right)$.

\subsection{Coupling Au NCs with PNIPAAm}

Mercaptopyridine-activated PNIPAAm was added to a stirred solution of luminescent $\mathrm{Au}$ NCs (10 mg in $10 \mathrm{~mL}$ of PBS buffer at $\mathrm{pH} 8.0)$. The mixed solution was stirred gently ( $300 \mathrm{rpm}$ ) for $24 \mathrm{~h}$ and then purified by a centrifugal filter (MWCO $10 \mathrm{kDa}$ ) to remove unreacted PNIPAAm and salts. After freeze-drying, the Au NCs-PNIPAAm conjugates were obtained.

\section{Results and Discussion}

\subsection{Relay Luminescence Enhancement with Aggregation-Induced Emission (AIE)}

In the present study, GSH-protected Au NCs were chosen as starting materials due to the following reasons: (i) GSH has various functional groups, such as thiol, amine, and carboxylic group. These functional groups can be used not only for the preparation of Au NCs, but also for the conjugation of other functional molecules; and (ii) GSH-protected Au NCs have high biocompatibility, which is a prerequisite for their applications in biomedicine [58-60]. Driven by these unique properties, GSH-protected luminescent Au NCs were synthesized according to the reported protocol [35]. To study the photothermal and thermosensitive properties of the 
as-synthesized luminescent Au NCs, it is important to functionalize the surface of the NCs with temperature-sensitive molecules. In this context, PNIPAAm is a good candidate because PNIPPAAm has a low critical solution temperature (LCST) of $32^{\circ} \mathrm{C}$. More interestingly, when the temperature is lower than LCST, it is hydrophilic (has good solubility in water), and when the temperature is higher than LCST, it becomes hydrophobic [61].

As a proof of concept, PNIPAAm was synthesized according to our reported protocol [62-68]. The as-generated mercaptothiazoline-activated PNIPAAm $\left(\mathrm{Mn}=9600 \mathrm{~g} \mathrm{~mol}^{-1}\right.$, $\mathrm{PDI}=1.2$, ca. monomer repeating units 85 ) was then conjugated to the primary amine on the surface of Au NCs (please refer to the experimental section for the detailed synthesis and characterizations, Figure S1). The resulting nanoconjugates (Au NCs-PNIPAAm) showed a significant change in hydrodynamic diameter from dynamic light scattering (DLS) analysis. Upon conjugation, the mean hydrodynamic diameter of the Au NCs with the polymer increased from $1.7 \pm 0.2 \mathrm{~nm}$ to $4.8 \pm 0.3 \mathrm{~nm}$ (Figure S2-S5). The corresponding zeta potentials of $\mathrm{Au}$ NCs and Au NCs-PNIPAAm are -7.6 and $-17.3 \mathrm{mV}$, respectively (Figure S6). The increase in the negative charge of Au NCs-PNIPAAm is attributed to the decrease in the number of amine groups from the GSH ligands, which form amide bonds with PNIPAAm. In addition, unlike Au NCs, a broad peak at $~ 400 \mathrm{~nm}$ can be clearly seen from the UV-vis absorption spectrum of Au NCs-PNIPAAm (Figure 1a), which also supports the conjugation of PNIPAAm on the cluster surface. The luminescence spectrum of $\mathrm{Au}$ NCs has a peak at $\sim 610 \mathrm{~nm}$ (Figure 1b, blue curve). The luminescence intensity is obviously enhanced ( 2 times) upon the conjugation with PNIPAAm (Figure 1b, red curve). In fact, this increase in luminescence depends on the coupling between PNIPAAm and Au NCs. In particular, the amide bond between PNIPAAm and GSH on the NC surface restricts intramolecular rotation and vibration by rigidifying the ligand shell on the cluster surface. As a result, luminescence decay through the non-radiative channels is reduced, thereby enhancing the luminescence of Au NCs in the solution.
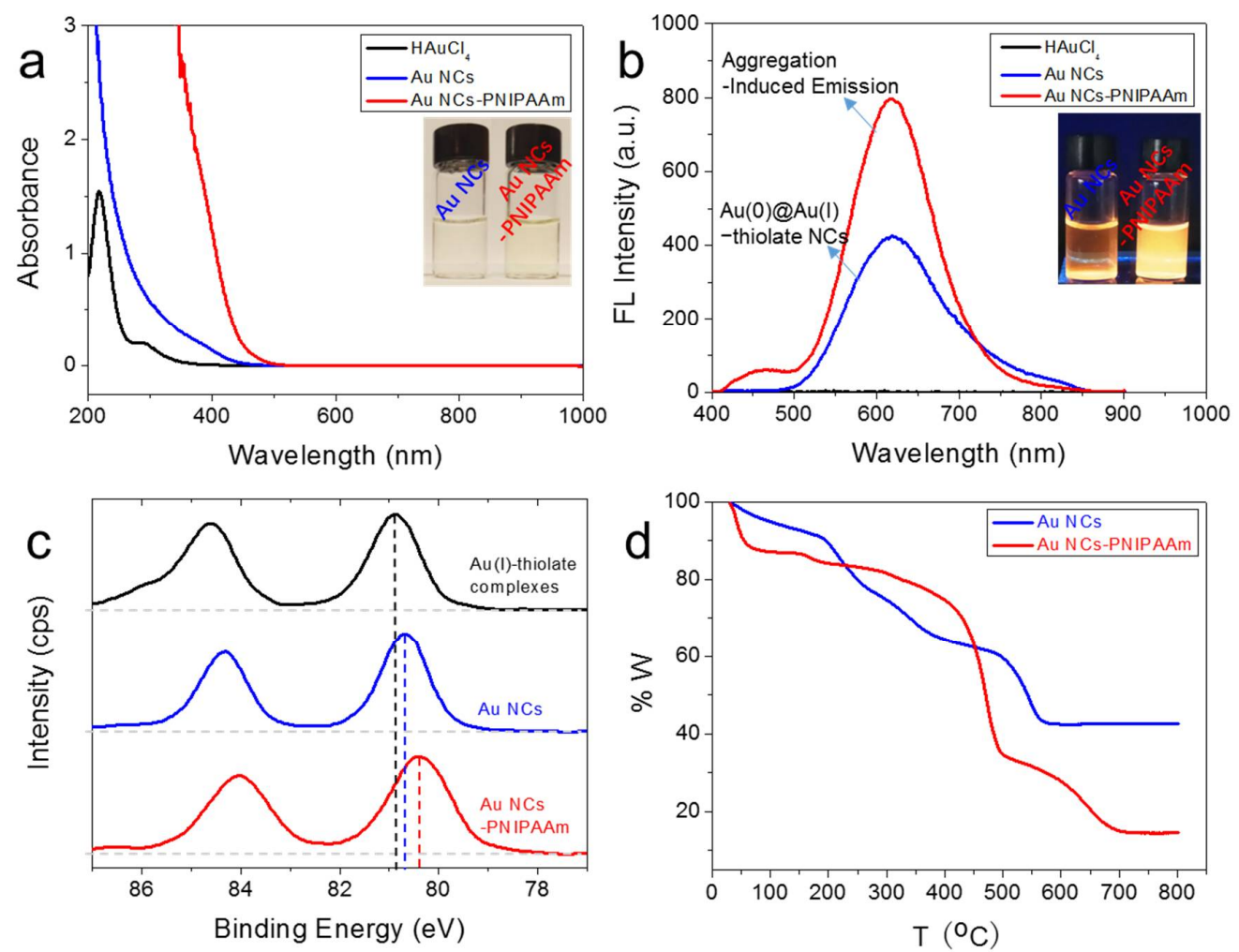

Figure 1. (a) UV-vis absorption and (b) photoemission spectra of $\mathrm{HAuCl}_{4}, \mathrm{Au} \mathrm{NCs}$, and $\mathrm{Au}$ NCs-PNIPAAm in aqueous solution $\left(\lambda_{\mathrm{ex}}=365 \mathrm{~nm}, \lambda_{\mathrm{em}}=610 \mathrm{~nm}\right.$ ). (Insets) Digital photos of luminescent Au NCs and Au NCs-PNIPAAm under ambient light and UV light. (c) Au 4f XPS spectra of Au-thiolate complexes, luminescent Au NCs, and Au NCs-PNIPAAm. (d) TGA curve of Au NCs and Au NCs-PNIPAAm. 
The as-synthesized Au NCs-PNIPAAm were further characterized by X-ray photoelectron spectroscopy (XPS) and thermogravimetric analysis (TGA) to study the chemical bond formation during the coupling process and the thermal stability of Au NCs-PNIPAAm, respectively. The XPS spectra in Figure 1c indicate that the oxidation state of Au in $\mathrm{Au}$ NCs-PNIPAAm (red line) is lower than that of Au-GSH complexes (black line) and luminescent $\mathrm{Au}$ NCs (blue line). The Au $4 \mathrm{f}$ spectrum of Au NCs-PNIPAAm has lower binding energies (84.1 and $80.4 \mathrm{eV}$ ) than luminescent Au NCs (84.3 and $80.7 \mathrm{eV}$ ) and Au-GSH complexes $(84.6$ and $80.9 \mathrm{eV})$. This is because the trithiocarbonate group of PNIPAAm has a strong combining capacity [11,69], which is capable of recombining $\mathrm{Au}(\mathrm{I})$ to form a higher conjugated structure. The thermal stability of Au NCs-PNIPAAm was assessed by TGA (Figure 1d). The degradation of PNIPAAm from the cluster surface was evident from the obvious weight loss after $400^{\circ} \mathrm{C}$. This result indicates that Au NCs-PNIPAAm was structurally robust even at elevated temperatures.

After successfully synthesizing Au NCs-PNIPAAm in aqueous solution, the effect of PNIPAAm ligand on the relay luminescence enhancement was studied (Figure 2a). In this case, we first monitored the synthesis process in a reaction time-dependent manner. As shown in Figure $2 b, c$, as time increased from 0 to $32 \mathrm{~h}$, the luminescence peak intensity at $\sim 610 \mathrm{~nm}$ (excitation wavelength at $365 \mathrm{~nm}$ ) increased monotonically to $16 \mathrm{~h}$ and stabilized after $24 \mathrm{~h}$, indicating that the combination process is step by step. The excited state lifetime decay profiles of the aqueous dispersion of Au NCs and Au NCs-PNIPAAm at $\lambda=610 \mathrm{~nm}$ $\left(\lambda_{\mathrm{ex}}=344 \mathrm{~nm}\right)$ indicate the predominance of microsecond lifetime components in both the luminescent Au NCs $(2.44 \mu \mathrm{s}, 75 \% ; 139 \mathrm{~ns}, 8.6 \%)$ and Au NCs-PNIPAAm $(2.87 \mu \mathrm{s}, 62 \%$; $107 \mathrm{~ns}, 4.2 \%$ ) (Figure 2d,e). The residuals of the two spectra are close to zero (Figure S7). The result corresponds to a relatively longer lifetime of Au NCs-PNIPAAm than Au NCs and explains the effect of relay luminescence enhancement. In particular, the PNIPAAm ligands were covalently attached to the surface of Au NCs and further induced the aggregation of Au NCs, which led to enhanced luminescence through RIR and RIV mechanisms. In addition, the trithiocarbonate group has a strong combining capacity and forms a larger conjugated structure through a recombining process with $\mathrm{Au}$. Therefore, we attributed this interesting relay luminescence enhancement to the mechanism of RIR and RIV with AIE characteristics.

\subsection{Relay Intensity Decrease with Temperature}

Considering the high luminescence of Au NCs, which is known to be sensitive to the environment, and the fact that the Au NCs were coupled with a thermoresponsive polymer, the thermoresponsive properties of Au NCs-PNIPAAm were studied. As shown in the digital photos of Figure 3b, the solutions of Au NCs-PNIPAAm gradually turned turbid with the increase of temperature at a constant conjugate concentration of $1 \mathrm{mg} \mathrm{mL}^{-1}$. In contrast, the corresponding luminescence was weakened, especially at temperatures above $32{ }^{\circ} \mathrm{C}$ (bottom row, Figure $3 \mathrm{~b}$ ). The opaque and weak luminescence of the solution strongly indicates the change of Au NCs-PNIPAAm with increasing temperature. As a proof of concept, when the temperature decreases, the luminescence of the corresponding Au NCsPNIPAAm solution returns to the original intensity (Figure S8). However, it is undeniable that the luminescence intensity is not as high as before, which also indirectly proves that the luminescence of Au NCs-PNIPAAm solution has good cycling characteristics. Furthermore, different luminescence intensities can be cycled multiple times with temperature changes (Figure S9). It is worth mentioning that the thermoresponsive nature of luminescent Au NCs-PNIPAAm upon aggregation was different from the previously described relay luminescence enhancement. 
a

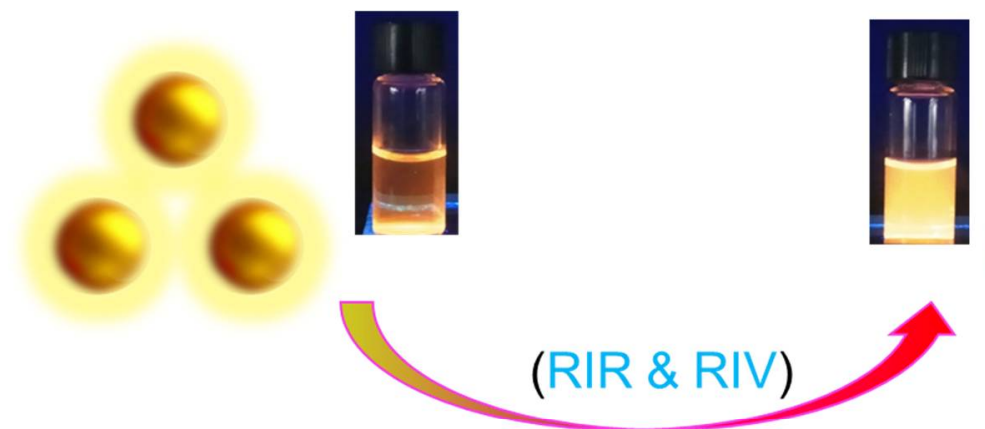

\section{Aggregation-Induced Emission}
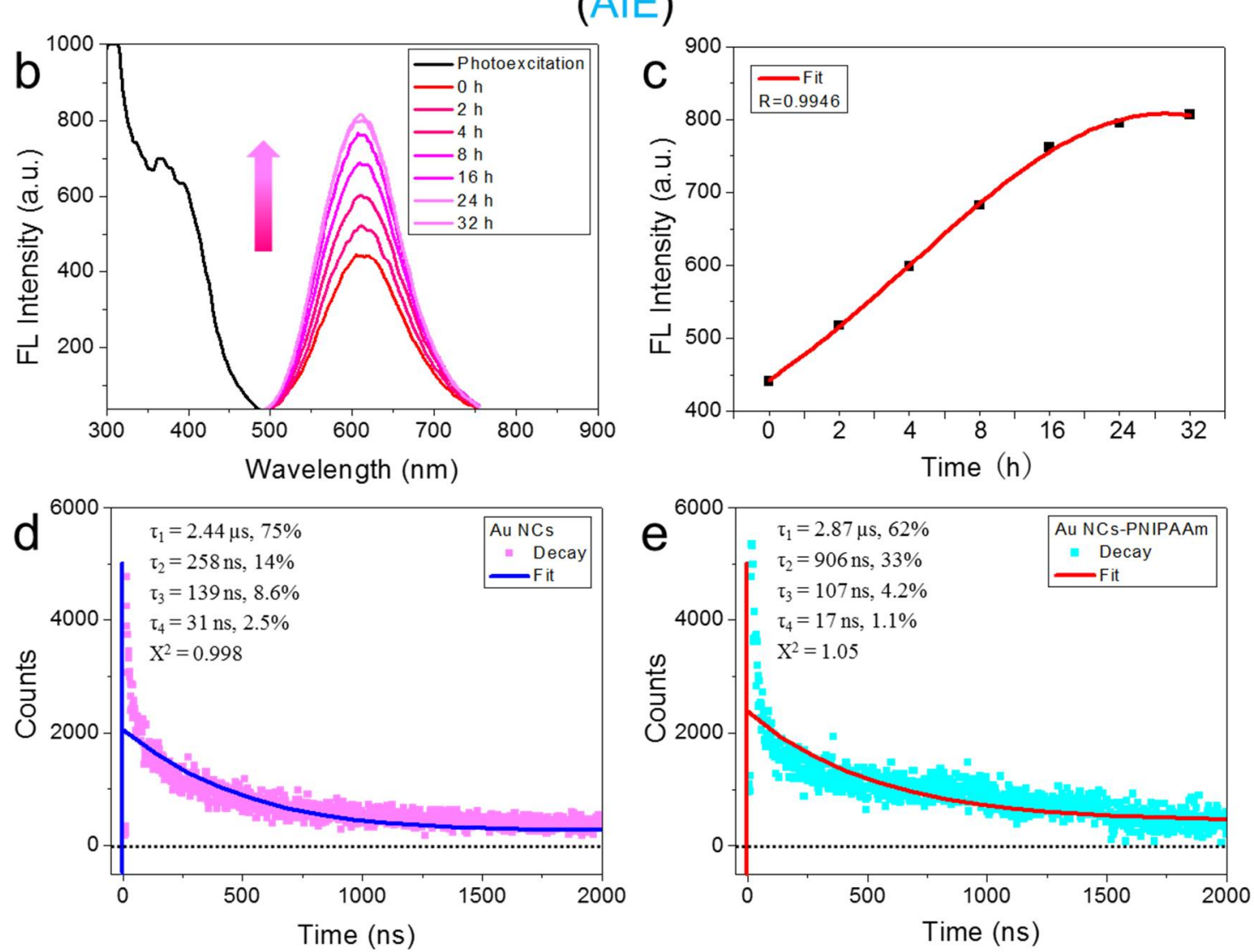

Figure 2. (a) Schematic illustration of the proposed mechanism for aggregation-induced emission (AIE), restriction of intramolecular rotation (RIR), and restriction of intramolecular vibration (RIV). (b) (Red lines) Photoemission spectra of the solution with different reaction times of the coupling process (the initiation of the reaction was defined as $0 \mathrm{~h}$ ). (Black line) Photoexcitation spectra of $\mathrm{Au}$ NCs-PNIPAAm after $0 \mathrm{~h}$ of the coupling reaction. The excitation wavelength of the photoemission spectra is $400 \mathrm{~nm}$ and the emission wavelength of the photoexcitation spectrum is $620 \mathrm{~nm}$. (c) The relationship between the luminescence intensity and the coupling time. (d,e) Photoluminescence decay profiles of the luminescent Au NCs and the as-synthesized Au NCs-PNIPAAm in water (0-2000 ns). 


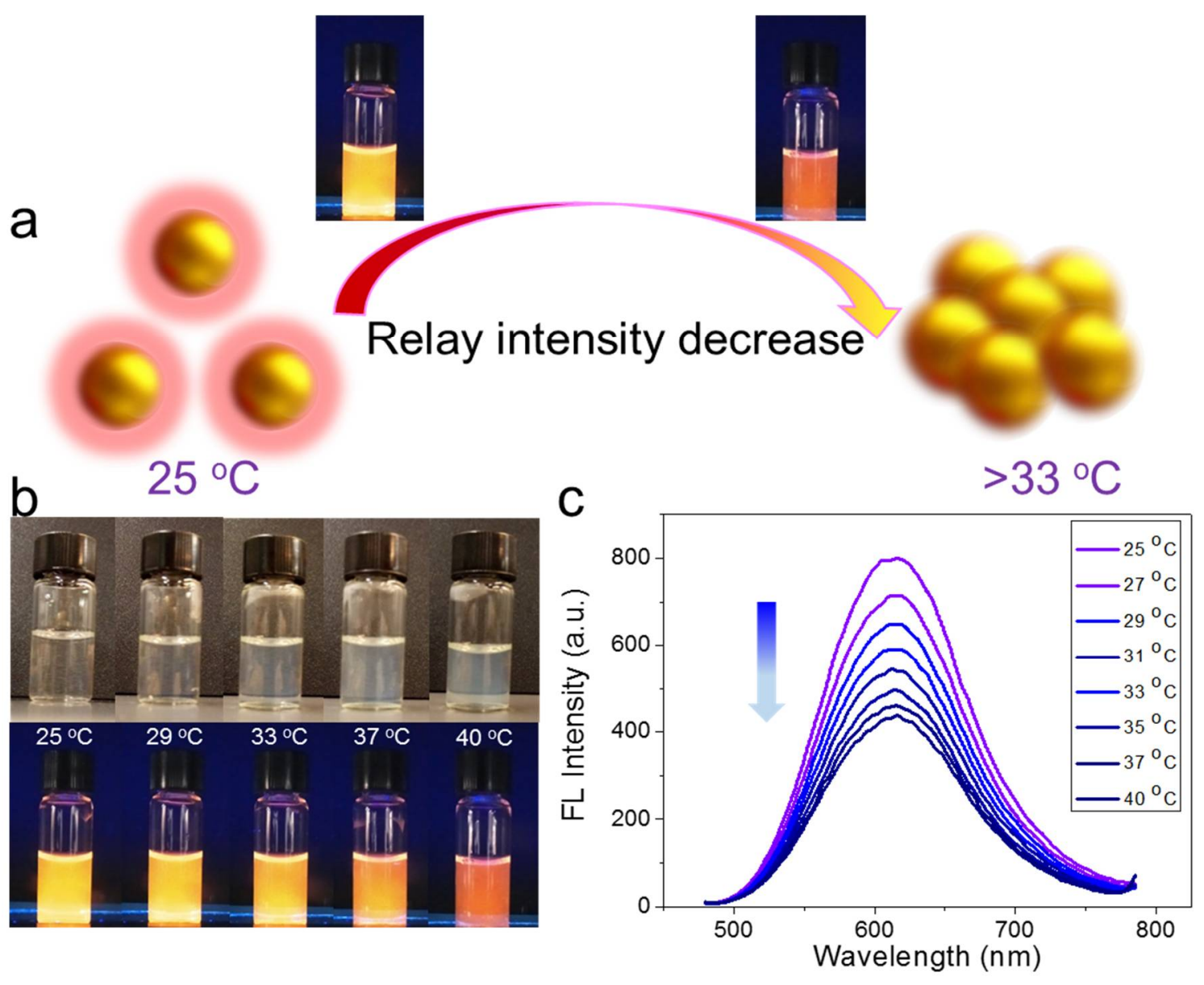

Figure 3. (a) Schematic illustration of the proposed mechanism for relay intensity decrease, where a higher temperature would induce the shrinkage of the thermosensitive polymer (PNIPAAm), leading to the weakened luminescence. (b) Digital photos of Au NCs-PNIPAAm $\left(1 \mathrm{mg} \mathrm{mL}^{-1}\right)$ in water with increasing temperature under visible (top row) and UV light (365 nm, bottom row). (c) The corresponding photoemission spectra of Au NCs-PNIPAAm at different temperatures (the excitation and emission wavelengths are 400 and $610 \mathrm{~nm}$, respectively).

Although in both cases aggregation plays an important role in the luminescence properties, the mechanism of temperature-induced luminescence changes seems to be consistent with the common intensity decrease with temperature. However, the temperature here causes changes in the optical properties of Au NCs, which is different from the conventional ACQ effect. To verify this, DLS measurements were performed, showing that the mean hydrodynamic diameter of Au NCs-PNIPAAm changed from $4.8 \pm 0.3 \mathrm{~nm}$ at room temperature to $68.7 \pm 1.0 \mathrm{~nm}$ at $37^{\circ} \mathrm{C}$ (Figure S10). In particular, the increase in the hydrophobicity of PNIPAAm will cause the aggregation of Au NCs-PNIPAAm, which may be the main reason for the decrease of luminescence. The corresponding luminescence spectra clearly showed that when the solution temperature increased from 25 to $40{ }^{\circ} \mathrm{C}$, the luminescence intensity of Au NCs-PNIPAAm gradually decreased (Figure 3c). The above results indicate that the change in temperature is crucial to the optical properties of $\mathrm{Au}$ NCs-PNIPAAm, that is, increasing temperature reduces the luminescence intensity of the composites. Therefore, this relay intensity decrease with temperature can provide a good platform to study the relay decay after the relay luminescence enhancement (Figure 3a).

\subsection{In-Situ Observations of Relay Intensity Decrease}

The relay properties provide a unique perspective for the functionalized Au NCsPNIPAAm to evaluate the optical properties of $\mathrm{Au} \mathrm{NC}$ derivatives. To quantitatively 
evaluate the changes in the relay intensity of the constructed Au NCs-PNIPAAm, we studied the self-assembly of Au NCs-PNIPAAm conjugates in solution. These conjugates can be used as building blocks of vesicles through a typical Pickering emulsion procedure. 2-ethyl-1-hexanol was used as the organic phase at an aqueous/organic volume fraction of 0.06. In the presence of the cross-linking agent glutaraldehyde (GA), the resulting vesicles can be transferred well into the aqueous solution without loss of structural integrity, which can be verified by luminescence microscope, confocal laser scanning microscope, transmission electron microscope (TEM), and atomic force microscope (AFM). The diameters of the constructed vesicles are in the range of 5-20 $\mu \mathrm{m}$ and their structures were found to be robust with non-aggregated status in aqueous solution evidenced by optical microscope (Figure 4a). Luminescence microscope images showed strong emission at the red channel, indicating that the as-prepared $\mathrm{Au}$ NCs-PNIPAAm has strong luminescence and high stability in aqueous solution (Figure S11). The optical and luminescence microscope images of the partially dried structures are composed of flexible ultrathin films that are visible to the naked eyes (Figure S12). TEM images of the vesicles suggest that the ultrathin films were at a different level, and the enlarged edges of the vesicles were continuous and integrated even under vacuum (Figure S13). In addition, through the section scanning model (Figure 4c,d), the AFM images of the vesicles showed different thicknesses, about $13 \mathrm{~nm}$ in different regions of the films, which is associated with the different level of the films.

To further reveal the structure of self-assembled vesicles, we studied the 3D structure in different ways. The 3D confocal laser scanning microscopy (CLSM) images (Figures 4b and S14) showed the hollow structures that are robust without loss of structural integrity. The 3D AFM images (Figure S15) also suggest that the height of the vesicles is about $90 \mathrm{~nm}$. By scanning the $\mathrm{XY}$ and $\mathrm{YZ}$ axes, the structure of the films can be clearly seen from the bottom and right images (Figure S16).
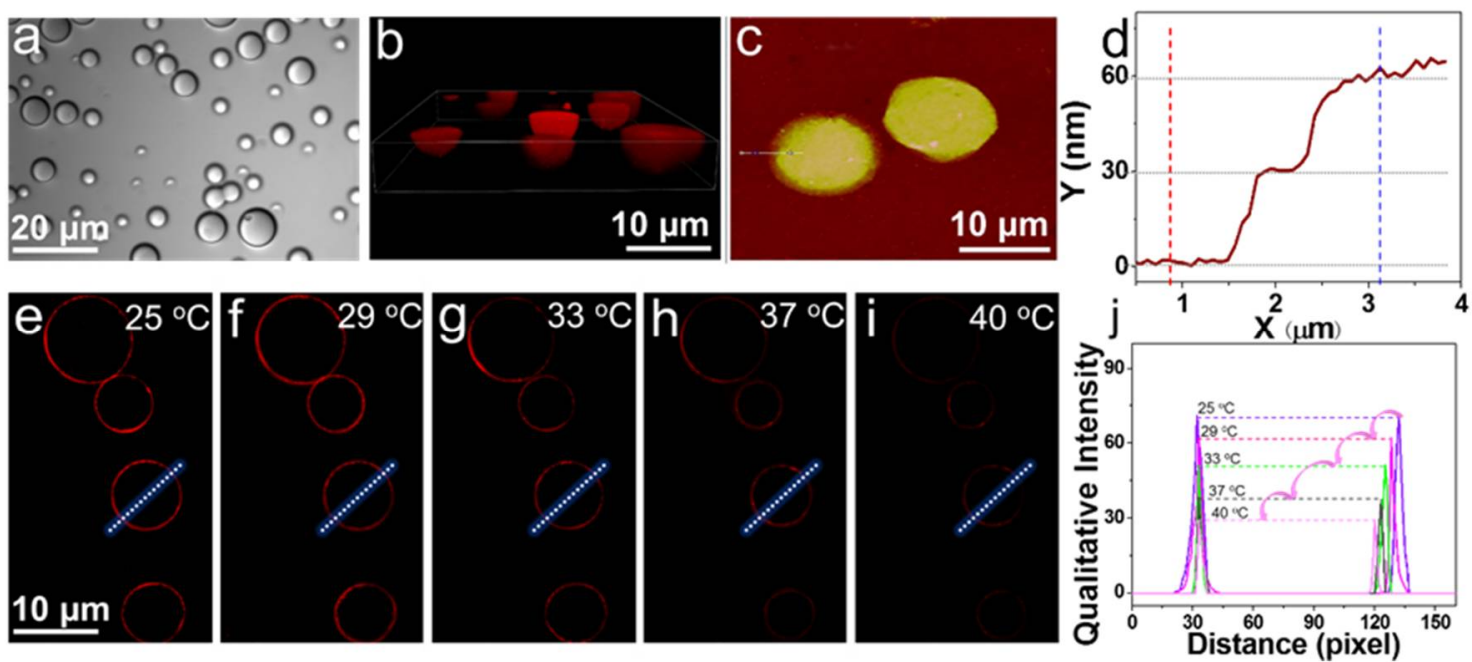

Figure 4. (a) Optical microscope image and (b) 3D CLSM image of Au NCs-PNIPAAm vesicles. (c) AFM images of the dry vesicles and (d) the corresponding section scanning of the films (red/blue line, the average value). (e-i) In-situ CLSM images of Au NCs-PNIPAAm vesicles from 25 to $40{ }^{\circ} \mathrm{C}$ showing the temperature-induced relay decrease in luminescence intensity accompanied by the decrease in diameter. (j) Corresponding intensity profiles and diameter variation for single vesicle shown in $(\mathbf{e}-\mathbf{i})$.

According to the difference in surface roughness, the films are structurally robust. More importantly, certain areas of the folded films are robust even when they are distorted to a great extent. Considering the unique thermosensitive characteristics of $\mathrm{Au}$ NCs-PNIPAAm, the above results suggest that the relay intensity is reduced due to temperature modulation. We also studied whether increasing the temperature will affect the surface roughness and the intact structure of the films. To confirm this, the corresponding 
roughness $(\mathrm{Ra})$ decreased from $2.00 \mathrm{~nm}\left(25^{\circ} \mathrm{C}\right)$ to $1.48 \mathrm{~nm}\left(37^{\circ} \mathrm{C}\right)$ without/with increasing temperature, which is associated with a temperature higher than LCST $\left(32{ }^{\circ} \mathrm{C}\right)$, indicating that the thermosensitive PNIPAAm remained attached to the films (Figure S17). Inspired by the above results, to further understand whether the temperature-induced optical property transition will affect the vesicles in the aqueous solution, we attempted to verify the continuous heating effects. Indeed, we found that the increase in temperature was also responsible for the reduction in size. As shown in Figure $4 \mathrm{e}-\mathrm{i}$, the temperature stimuli behavior was monitored using CLSM by measuring individual water-dispersed vesicles thermally cycling from 25 to $33^{\circ} \mathrm{C}$. As the temperature increased, the phase change of PNIPAAm resulted in about $58.6 \%$ of luminescence intensity loss and $9 \%$ of decrease in the vesicle diameter (Figure 4j). The generated Au NCs-PNIPAAm vesicles showed obvious changes in optical properties against temperature-induced relay intensity decrease.

\section{Conclusions}

Luminescent Au NCs were functionalized with thermosensitive PNIPAAm through covalent bonds, showing two interesting phenomena, namely, relay luminescence enhancement by AIE and temperature-induced relay intensity decrease. The restriction of intramolecular rotation and vibration and the formation of higher conjugated structures, both induced by PNIPAAm, resulted in relay luminescence enhancement. On the other hand, PNIPAAm on the cluster surface became denser at a temperature higher than LCST, resulting in relay intensity decrease. This relay intensity decrease can also be achieved by assembling Au NCs-PNIPAAm into vesicles. The findings revealed in this study may help to explore the optical properties of metal NCs in various applications.

Supplementary Materials: The following are available online at https: / www.mdpi.com/article / 10.3390/nano12050777/s1, Figure S1. ${ }^{1} \mathrm{H}$ NMR spectrum of the end-capped mercaptothiazolineactivated PNIPAAm (Mn $9600 \mathrm{~g} \mathrm{~mol}^{-1}$ ) in $\mathrm{CDCl}_{3}, 400 \mathrm{MHz}$; Figure S2: DLS profiles of the aqueous Au NCs (black, $1.7 \pm 0.2 \mathrm{~nm}$ ) and Au NCs-PNIPAAm (red, $4.8 \pm 0.3 \mathrm{~nm}$ ) in water at room temperature; Figure S3. A representative TEM image of the as-synthesized luminescent Au NCs; Figure S4. Particle size distribution of the aqueous Au NCs $(1.7 \pm 0.2 \mathrm{~nm})$ in water at room temperature; Figure S5. Particle size distribution of the aqueous Au NCs-PNIPAAm $(4.8 \pm 0.3 \mathrm{~nm})$ in water at room temperature; Figure S6. Zeta potential measurement for Au NCs (purple, $-7.6 \mathrm{mV}$ ) and Au NCs-PNIPAAm (red, $-17.3 \mathrm{mV}$ ) in water at room temperature; Figure S7. $(\mathrm{a}, \mathrm{b})$ Photoluminescence decay profiles of $\mathrm{Au}$ NCs and Au NCs-PNIPAAm (0-8000 ns) in water. (c, d) Residuals of the corresponding lifetime decay profiles of Au NCs and Au NCs-PNIPAAm; Figure S8. Digital photos of Au NCs-PNIPAAm $\left(1 \mathrm{mg} \mathrm{mL}^{-1}\right)$ in water with decreasing temperature under UV light (365 nm); Figure S9. Luminescence intensity of five complete reversible cycles as followed by alternate low temperature $\left(25^{\circ} \mathrm{C}\right)$ and high temperature $\left(37^{\circ} \mathrm{C}\right)$; Figure S10. Size distribution of Au NCs-PNIPAAm below (red, $4.8 \pm 0.3 \mathrm{~nm}$, $25^{\circ} \mathrm{C}$ ) and above (blue, $68.7 \pm 1 \mathrm{~nm}, 37^{\circ} \mathrm{C}$ ) the LCST of PNIPAAm; Figure S11. Representative fluorescence microscope image of the Au NCs-PNIPAAm vesicles; Figure S12. (a) Optical microscope and (b) fluorescence microscope image of dry Au NCs-PNIPAAm vesicles; Figure S13. Transmission electron microscope (TEM) images of Au NCs-PNIPAAm vesicles (left), and the enlarged region (right), showing the structure of the films at different levels; Figure S14. CLSM images of the 3D structure of the Au NCs-PNIPAAm vesicles; Figure S15. Atomic force microscope (AFM) images of $\mathrm{Au}$ NCs-PNIPAAm vesicles, and the clear stereoscopic structure showing the distinguishing height from the edge to center; Figure S16. 3D scanning model of the XY and YZ axes, showing the continuous and robust films of the constructed Au NCs-PNIPAAm vesicles; Figure S17. Atomic force microscope (AFM) images of Au NCs-PNIPAAm vesicles: (a) below and (b) above the LCST, showing the different roughnesses of the selected regions a $(\mathrm{Rq}=2.54 \mathrm{~nm}, \mathrm{Ra}=2 \mathrm{~nm})$ and $\mathrm{b}(\mathrm{Rq}=1.87 \mathrm{~nm}, \mathrm{Ra}$ $=1.48 \mathrm{~nm}$ ). The following data corresponds to the roughness parameter of the film.

Author Contributions: P.Z. conceptualization, investigation, data analysis, formal analysis, investigation, writing —original draft; N.G. methodology, software, visualization, validation, running calculations; T.C. software, visualization, validation; X.L. conceptualization, formal analysis; X.H. supervision, correction, writing-review \& editing. All authors have read and agreed to the published version of this manuscript. 
Funding: This work was financially supported by the Industry-University-Research (IUR) Project of Ministry of Education (202002292014); the Science and Technology Project of Henan Province (212102310511); the Scientific Research Fund of Henan Provincial Education Department (21A150032), Ministry of Education, Singapore (under Research Grant R-279-000-580-112 and R-279-000-538-114); National Natural Science Foundation of China $(21871069,51873050)$; and China Scholarship Council.

Institutional Review Board Statement: Not applicable.

Informed Consent Statement: Not applicable.

Data Availability Statement: Data is contained within the article or in Electronic Supporting Information (ESI).

Conflicts of Interest: The authors declare no conflict of interest.

\section{References}

1. Jadzinsky, P.D.; Calero, G.; Ackerson, C.J.; Bushnell, D.A.; Kornberg, R.D. Structure of a Thiol Monolayer-Protected Gold Nanoparticle at $1.1 \AA$ Resolution. Science 2007, 318, 430-433. [CrossRef] [PubMed]

2. Yi, C.; Tofanelli, M.A.; Ackerson, C.J.; Knappenberger, K.L. Optical Properties and Electronic Energy Relaxation of Metallic Au144(SR)60 Nanoclusters. J. Am. Chem. Soc. 2013, 135, 18222-18228. [CrossRef] [PubMed]

3. Jin, R.; Zeng, C.; Zhou, M.; Chen, Y. Atomically Precise Colloidal Metal Nanoclusters and Nanoparticles: Fundamentals and Opportunities. Chem. Rev. 2016, 116, 10346-10413. [CrossRef] [PubMed]

4. Chakraborty, I.; Pradeep, T. Atomically Precise Clusters of Noble Metals: Emerging Link between Atoms and Nanoparticles. Chem. Rev. 2017, 117, 8208-8271. [CrossRef]

5. Niihori, Y.; Shima, D.; Yoshida, K.; Hamada, K.; Nair, L.V.; Hossain, S.; Kurashige, W.; Negishi, Y. High-performance liquid chromatography mass spectrometry of gold and alloy clusters protected by hydrophilic thiolates. Nanoscale 2018, 10, 1641-1649. [CrossRef]

6. Lu, Y.; Chen, W. Sub-nanometre sized metal clusters: From synthetic challenges to the unique property discoveries. Chem. Soc. Rev. 2012, 41, 3594-3623. [CrossRef]

7. Qian, H.; Zhu, M.; Wu, Z.; Jin, R. Quantum Sized Gold Nanoclusters with Atomic Precision. Acc. Chem. Res. 2012, 45, 1470-1479. [CrossRef]

8. Saha, K.; Agasti, S.S.; Kim, C.; Li, X.; Rotello, V.M. Gold Nanoparticles in Chemical and Biological Sensing. Chem. Rev. 2012, 112, 2739-2779. [CrossRef]

9. Chen, Y.S.; Kamat, P.V. Glutathione-Capped Gold Nanoclusters as Photosensitizers. Visible Light-Induced Hydrogen Generation in Neutral Water. J. Am. Chem. Soc. 2014, 136, 6075-6082. [CrossRef]

10. Sajanlal, P.R.; Sreeprasad, T.S.; Samal, A.K. Pradeep, T. Anisotropic nanomaterials: Structure, growth, assembly, and functions. Nano Rev. 2011, 2, 5883. [CrossRef]

11. Häkkinen, H. The gold-sulfur interface at the nanoscale. Nat. Chem. 2012, 4, 443-445. [CrossRef]

12. Takano, S.; Yamazoe, S.; Koyasu, K.; Tsukuda, T. Slow-Reduction Synthesis of a Thiolate-Protected One-Dimensional Gold Cluster Showing an Intense Near-Infrared Absorption. J. Am. Chem. Soc. 2015, 137, 7027-7030. [CrossRef]

13. Gan, Z.; Lin, Y.; Luo, L.; Han, G.; Liu, W.; Liu, Z.; Yao, C.; Weng, L.; Liao, L.; Chen, J.; et al. Fluorescent Gold Nanoclusters with Interlocked Staples and a Fully Thiolate-Bound Kernel. Angew. Chem. Int. Ed. 2016, 55, 11567-11571. [CrossRef]

14. Kurashige, W.; Niihori, Y.; Sharma, S.; Negishi, Y. Precise synthesis, functionalization and application of thiolate-protected gold clusters. Coord. Chem. Rev. 2016, 320, 238-250. [CrossRef]

15. Yu, H.; Rao, B.; Jiang, W.; Yang, S.; Zhu, M. The photoluminescent metal nanoclusters with atomic precision. Coord. Chem. Rev. 2019, 378, 595-617. [CrossRef]

16. Zheng, J.; Zhang, C.; Dickson, R.M. Highly Fluorescent, Water-Soluble, Size-Tunable Gold Quantum Dots. Phys. Rev. Lett. 2004, 93, 077402. [CrossRef]

17. He, W.; Zhou, Z.; Han, Z.; Li, S.; Zhou, Z.; Ma, L.; Zang, S. Ultrafast Size Expansion and Turn-On Luminescence of Atomically Precise Silver Clusters by Hydrogen Sulfide. Angew. Chem. Int. Ed. 2021, 133, 8586-8590. [CrossRef]

18. Jain, P.K.; Huang, X.; El-Sayed, I.H.; El-Sayed, M.A. Noble Metals on the Nanoscale: Optical and Photothermal Properties and Some Applications in Imaging, Sensing, Biology. Acc. Chem. Res. 2008, 41, 1578-1586. [CrossRef]

19. Shang, L.; Dörlich, R.M.; Brandholt, S.; Schneider, R.; Trouillet, V.; Bruns, M.; Gerthsen, D.; Nienhaus, G.U. Facile preparation of water-soluble fluorescent gold nanoclusters for cellular imaging applications. Nanoscale 2011, 3, 2009-2014. [CrossRef]

20. Xiao, Y.; Wu, Z.; Yao, Q.; Xie, J. Luminescent metal nanoclusters: Biosensing strategies and bioimaging applications. Aggregate 2021, 2, 114-132. [CrossRef]

21. Liu, Z.; Wu, Z.; Yao, Q.; Cao, Y.; Chai, O.J.H.; Xie, J. Correlations between the fundamentals and applications of ultrasmall metal nanoclusters: Recent advances in catalysis and biomedical applications. Nano Today 2021, 36, 101053. [CrossRef]

22. Zheng, K.; Xie, J. Engineering ultrasmall metal nanoclusters as promising theranostic agents. Trends Chem. 2020, 2, 665-679. [CrossRef]

23. Jin, R. Quantum sized, thiolate-protected gold nanoclusters. Nanoscale 2010, 2, 343-362. [CrossRef] 
24. Wu, Z.; Jin, R. On the Ligand's Role in the Fluorescence of Gold Nanoclusters. Nano Lett. 2010, 10, 2568-2573. [CrossRef] [PubMed]

25. Pyo, K.; Thanthirige, V.D.; Kwak, K.; Pandurangan, P.; Ramakrishna, G.; Lee, D. Ultrabright Luminescence from Gold Nanoclusters: Rigidifying the Au(I)-Thiolate Shell. J. Am. Chem. Soc. 2015, 137, 8244-8250. [CrossRef]

26. Yau, S.H.; Varnavski, O.; Goodson, T. An Ultrafast Look at Au Nanoclusters. Acc. Chem. Res. 2013, 46, 1506-1516. [CrossRef]

27. Liu, J.; Duchesne, P.N.; Yu, M.; Jiang, X.; Ning, X.; Vinluan, R.D.; Zhang, P.; Zheng, J. Luminescent Gold Nanoparticles with Size-Independent Emission. Angew. Chem. Int. Ed. 2016, 55, 8894-8898. [CrossRef]

28. Weerawardene, K.L.D.M.; Aikens, C.M. Theoretical Insights into the Origin of Photoluminescence of Au25(SR)18-Nanoparticles J. Am. Chem. Soc. 2016, 138, 11202-11210. [CrossRef]

29. Weerawardene, K.L.D.M.; Guidez, E.B.; Aikens, C.M. Photoluminescence Origin of Au38(SR)24 and Au22(SR)18 Nanoparticles: A Theoretical Perspective. J. Phys. Chem. C 2017, 121, 15416-15423. [CrossRef]

30. Zheng, J.; Zhou, C.; Yu, M.; Liu, J. Different Sized Luminescent Gold Nanoparticles. Nanoscale 2012, 4, 4073-4083. [CrossRef]

31. Yahia-Ammar, A.; Sierra, D.; Mérola, F.; Hildebrandt, N.; Le Guével, X. Self-Assembled Gold Nanoclusters for Bright Fluorescence Imaging and Enhanced Drug Delivery. ACS Nano 2016, 10, 2591-2599. [CrossRef] [PubMed]

32. Wang, S.; Meng, X.; Das, A.; Li, T.; Song, Y.; Cao, T.; Zhu, X.; Zhu, M.; Jin, R. A 200-fold Quantum Yield Boost in the Photoluminescence of Silver-Doped agxau25-x Nanoclusters: The 13th Silver Atom Matters. Angew. Chem. Int. Ed. 2014, 53, 2376-2380. [CrossRef] [PubMed]

33. Wu, Z.; Yao, Q.; Zang, S.; Xie, J. Aggregation-induced emission in luminescent metal nanoclusters. Natl. Sci. Rev. 2021, 8, nwaa208. [CrossRef] [PubMed]

34. Wu, Z.; Yao, Q.; Chai, O.J.H.; Ding, N.; Xu, W.; Zang, S.; Xie, J. Unraveling the impact of gold (I)-thiolate motifs on the aggregation-induced emission of gold nanoclusters. Angew. Chem. Int. Ed. 2020, 59, 9934-9939. [CrossRef]

35. Luo, Z.; Yuan, X.; Yu, Y.; Zhang, Q.; Leong, D.T.; Lee, J.Y.; Xie, J. From Aggregation-Induced Emission of Au(I)-Thiolate Complexes to Ultrabright $\mathrm{Au}(0) @ \mathrm{Au}(\mathrm{I})$-Thiolate Core-Shell Nanoclusters. J. Am. Chem. Soc. 2012, 134, 16662-16670. [CrossRef]

36. Wang, M.; Zhang, G.; Zhang, D.; Zhu, D.; Tang, B.Z. Fluorescent bio/chemosensors based on silole and tetraphenylethene luminogens with aggregation-induced emission feature. J. Mater. Chem. 2010, 20, 1858-1867. [CrossRef]

37. Hong, Y.; Lam, J.W.Y.; Tang, B.Z. Aggregation-induced emission. Chem. Soc. Rev. 2011, 40, 5361-5388. [CrossRef]

38. Dou, X.; Yuan, X.; Yu, Y.; Luo, Z.; Yao, Q.; Leong, D.T.; Xie, J. Lighting up thiolated Au@Ag nanoclusters via aggregation-induced emission. Nanoscale 2014, 6, 157-161. [CrossRef]

39. Niu, C.; Liu, Q.; Shang, Z.; Zhao, L.; Ouyang, J. Dual-emission fluorescent sensor based on AIE organic nanoparticles and Au nanoclusters for the detection of mercury and melamine. Nanoscale 2015, 7, 8457-8465. [CrossRef]

40. Jia, X.; Yang, X.; Li, J.; Li, D.; Wang, E. Stable Cu nanoclusters: From an aggregation-induced emission mechanism to biosensing and catalytic applications. Chem. Commun. 2014, 50, 237-239. [CrossRef]

41. Wu, Z.; Yao, Q.; Liu, Z.; Xu, H.; Guo, P.; Liu, L.; Han, Y.; Zhang, K.; Lu, Z.; Li, X.; et al. Multiscale Assembly of [AgS 4 ] Tetrahedrons into Hierarchical Ag-S Networks for Robust Photonic Water. Adv. Mater. 2021, 33, 2006459. [CrossRef] [PubMed]

42. Mei, J.; Hong, Y.; Lam, J.W.Y.; Qin, A.; Tang, Y.; Tang, B.Z. Aggregation-induced emission: The whole is more brilliant than the parts. Adv. Mater. 2014, 26, 5429-5479. [CrossRef] [PubMed]

43. Wang, H.; Zhao, E.; Lam, J.W.Y.; Tang, B.Z. AIE luminogens: Emission brightened by aggregation. Mater. Today 2015, 18, 365-377. [CrossRef]

44. Mei, J.; Leung, N.L.C.; Kwok, R.T.K.; Lam, J.W.Y.; Tang, B.Z. Aggregation-Induced Emission: Together We Shine, United We Soar! Chem. Rev. 2015, 115, 11718-11940. [CrossRef]

45. Qian, H.; Cousins, M.E.; Horak, E.H.; Wakefield, A.; Liptak, M.D.; Aprahamian, I. Suppression of Kasha's rule as a mechanism for fluorescent molecular rotors and aggregation-induced emission. Nat. Chem. 2016, 9, 83-87. [CrossRef]

46. Guo, J.; Li, X.; Nie, H.; Luo, W.; Hu, R.; Qin, A.; Zhao, Z.; Su, S.-J.; Tang, B.Z. Robust Luminescent Materials with Prominent Aggregation-Induced Emission and Thermally Activated Delayed Fluorescence for High-Performance Organic Light-Emitting Diodes. Chem. Mater. 2017, 29, 3623-3631. [CrossRef]

47. Feng, G.; Kwok, R.T.K.; Tang, B.Z.; Liu, B. Functionality and versatility of aggregation-induced emission luminogens. Appl. Phys. Rev. 2017, 4, 021307. [CrossRef]

48. Ding, D.; Li, K.; Liu, B.; Tang, B.Z. Bioprobes Based on AIE Fluorogens. Acc. Chem. Res. 2013, 46, 2441-2453. [CrossRef]

49. Song, X.; Zhu, W.; Ge, X.; Li, R.; Li, S.; Chen, X.; Song, J.; Xie, J.; Chen, X.; Yang, H. A New Class of NIR-II Gold Nanocluster-Based Protein Biolabels for In Vivo Tumor-Targeted Imaging. Angew. Chem. Int. Ed. 2021, 60, 1306-1312. [CrossRef]

50. Liu, H.; Hong, G.; Luo, Z.; Chen, J.; Chang, J.; Gong, M.; He, H.; Yang, J.; Yuan, X.; Li, L.; et al. Atomic-precision gold clusters for NIR-II imaging. Adv. Mater. 2019, 31, 1901015. [CrossRef]

51. Bao, Y.; Guégain, E.; Nicolas, V.; Nicolas, J. Fluorescent polymer prodrug nanoparticles with aggregation-induced emission (AIE) properties from nitroxide-mediated polymerization. Chem. Commun. 2017, 53, 4489-4492. [CrossRef]

52. Kwok, R.T.K.; Leung, C.W.T.; Lam, J.W.Y.; Tang, B.Z. Biosensing by luminogens with aggregation-induced emission characteristics. Chem. Soc. Rev. 2015, 44, 4228-4238. [CrossRef]

53. Zhelev, Z.; Ohba, H.; Bakalova, R. Single Quantum Dot-Micelles Coated with Silica Shell as Potentially Non-Cytotoxic Fluorescent Cell Tracers. J. Am. Chem. Soc. 2006, 128, 6324-6325. [CrossRef] 
54. Bakalova, R.; Zhelev, Z.; Aoki, I.; Ohba, H.; Imai, Y.; Kanno, I. Silica-Shelled Single Quantum Dot Micelles as Imaging Probes with Dual or Multimodality. Anal. Chem. 2006, 78, 5925-5932. [CrossRef]

55. Tang, C.W.; VanSlyke, S.A. Organic electroluminescent diodes. Appl. Phys. Lett. 1987, 51, 913-915. [CrossRef]

56. Shustova, N.B.; McCarthy, B.D.; Dincă, M. Turn-On Fluorescence in Tetraphenylethylene-Based Metal-Organic Frameworks: An Alternative to Aggregation-Induced Emission. J. Am. Chem. Soc. 2011, 133, 20126-20129. [CrossRef]

57. Zhan, R.; Pan, Y.; Manghnani, P.N.; Liu, B. AIE Polymers: Synthesis, Properties, and Biological Applications. Macromol. Biosci. 2017, 17, 1600433. [CrossRef]

58. Yu, Y.; Luo, Z.; Chevrier, D.M.; Leong, D.T.; Zhang, P.; Jiang, D.; Xie, J. Identification of a Highly Luminescent Au $22(S G)_{18}$ Nanocluster. J. Am. Chem. Soc. 2014, 136, 1246-1249. [CrossRef]

59. Yu, Y.; Yao, Q.; Chen, T.; Lim, G.X.; Xie, J. The Innermost Three Gold Atoms Are Indispensable to Maintain the Structure of the $\mathrm{Au}_{18}(\mathrm{SR})_{14}$ Cluster. J. Phys. Chem. C 2016, 120, 22096-22102. [CrossRef]

60. Song, X.; Goswami, N.; Yang, H.; Xie, J. Functionalization of metal nanoclusters for biomedical applications. Analyst 2016, 141, 3126-3140. [CrossRef]

61. Ono, Y.; Shikata, T. Hydration and Dynamic Behavior of Poly(N-isopropylacrylamide)s in Aqueous Solution: A Sharp Phase Transition at the Lower Critical Solution Temperature. J. Am. Chem. Soc. 2006, 128, 10030-10031. [CrossRef]

62. Zhou, P.; Liu, X.; Wu, G.; Wen, P.; Wang, L.; Huang, Y.; Huang, X. Programmable Modulation of Membrane Permeability of Proteinosome upon Multiple Stimuli Responses. ACS Macro Lett. 2016, 5, 961-966. [CrossRef]

63. Zhou, P.; Wu, S.; Liu, X.; Hegazy, M.; Wu, G.; Huang, X. Multifunctional and Programmable Modulated Interface Reactions on Proteinosomes. ACS Appl. Mater. Interfaces 2018, 10, 38565-38573. [CrossRef] [PubMed]

64. Hegazy, M.; Zhou, P.; Wu, G.; Wang, L.; Rahoui, N.; Huang, X.; Huang, Y. Construction of polymer coated core-shell magnetic mesoporous silica nanoparticles with triple responsive drug delivery. Polym. Chem. 2017, 8, 5852-5864. [CrossRef]

65. Liu, X.; Zhou, P.; Huang, Y.; Li, M.; Huang, X.; Mann, S. Hierarchical Proteinosomes for Programmed Release of Multiple Components. Angew. Chem. Int. Ed. 2016, 55, 7095-7100. [CrossRef] [PubMed]

66. Zhou, P.; Wu, S.; Hegazy, M.; Li, H.; Xu, X.; Lu, H.; Huang, X. Engineered borate ester conjugated protein-polymer nanoconjugates for $\mathrm{pH}$-responsive drug delivery. Mater. Sci. Eng. C 2019, 104, 109914. [CrossRef]

67. Wu, G.; Liu, X.; Zhou, P.; Wang, L.; Hegazy, M.; Huang, X.; Huang, Y. A facile approach for the reduction of 4-nitrophenol and degradation of congo red using gold nanoparticles or laccase decorated hybrid inorganic nanoparticles/polymerbiomacromolecules vesicles. Mater. Sci. Eng. C 2019, 94, 524-533. [CrossRef] [PubMed]

68. Wu, G.; Liu, X.; Zhou, P.; Xu, Z.; Hegazy, M.; Huang, X.; Huang, Y. The construction of thiol-functionalized DNAsomes with small molecules response and protein release. Mater. Sci. Eng. C 2019, 99, 1153-1163. [CrossRef]

69. Boles, M.A.; Ling, D.; Hyeon, T.; Talapin, D.V. The surface science of nanocrystals. Nat. Mater. 2016, 15, 141-153. [CrossRef] 